\title{
Treino Musical e Capacidade da Memória Operacional em Crianças Iniciantes, Veteranas e Sem Conhecimentos Musicais
}

\author{
Musical Training and Working Memory Span in Beginners, Veterans \\ and with no Musical Knowledge Children
}

\author{
Fabiana Silva Ribeiro* \& Flávia Heloísa Dos Santos \\ Universidade Estadual Paulista Júlio de Mesquita Filho, Assis, Brasil
}

\begin{abstract}
Resumo
A memória operacional e a atividade musical ativam áreas encefálicas recíprocas e homólogas, contudo não há evidências se o treino musical pode ampliar a capacidade da memória operacional. Objetivo: Avaliar o desempenho do treino musical sob a memória operacional em crianças de 9 e 10 anos de idade, praticantes de treino musical e sem experiência musical. Participantes: Crianças Iniciantes $(n=20)$, Veteranas $(n=20)$ e Grupo Controle $(n=20)$. Materiais: Instrumentos computadorizados para avaliação da memória operacional. Resultados: Crianças veteranas apresentaram melhores pontuações no BCPR (Teste de Repetição de Pseudopalavras para crianças brasileiras) e em subtestes da AWMA (Avaliação Automatizada da Memória Operacional). Conclusão: O treino musical parece ter contribuído para o desenvolvimento da memória operacional em crianças veteranas no programa de treino musical.

Palavras-chave: AWMA, memória operacional, treino musical, ensino da música.
\end{abstract}

\begin{abstract}
Working memory and musical activity activate reciprocal and homologues brain areas. However, there is a lack of evidence if musical training can amplify the working memory span. Objective: To assess the performance of musical training on working memory in children aged 9 and 10 years old who regularly have musical training or with no musical training at all. Participants: Beginners $(n=20)$, Veterans $(n=20)$ and a Control Group $(n=20)$. Materials: Computerized instruments to assess working memory. Results: Veteran children presented higher scores in BCPR (Brazilian Children's Test of Pseudoword Repetition) and in specific tasks of AWMA (Automated Working Memory Assessment). Conclusion: Musical training seems to have contributed for working memory development in veteran children under musical training programs.

Keywords: AWMA, working memory, musical training, music teaching.
\end{abstract}

A memória operacional pode ser definida como um sistema complexo, que possui a capacidade de reter e manipular informações por curtos períodos de tempo (Baddeley \& Hitch, 1974). Esse sistema possui atualmente quatro componentes. Um deles é a alça fonológica que possui a função de armazenar e manipular informações baseadas na fala, e recebe informação tanto por via direta (apresentação auditiva) quanto por via indireta (apresentação visual). Outro componente é o esboço visuoespacial que armazena informações visuais e espaciais, como lembrar formas e cores, locais e veloci-

\footnotetext{
* Endereço para correspondência: Departamento de Psicologia Experimental e do Trabalho, Universidade Estadual Paulista, Laboratório de Neuropsicologia, Avenida Dom Antônio, 2100, Assis, SP, Brasil 19806-900. Tel.: + 5518 3302-5902. E-mail: flaviahs@assis.unesp.br Resultados parciais deste estudo foram apresentados no III Congresso Multidisciplinar de Transtornos de Aprendizagem e Reabilitação (São Paulo, 2010), tendo recebido o Prêmio de Melhor Poster.
}

dade de objetos no espaço. Este também está envolvido em tarefas que necessitam planejamento de movimento no espaço (Baddeley, 2000).

Um terceiro componente, o executivo central, é o sistema controlador da atenção que não exibe especificidade modal, possui capacidade atencional limitada e é supostamente responsável pelo processamento de tarefas cognitivas (Baddeley, 1992). Está relacionado à organização de aprendizagem e planejamento das informações (Repovš \& Baddeley, 2006). E finalmente o retentor episódico, que integra e armazena informações fonológica, visual, espacial e memória de longo prazo, temporariamente em uma representação episódica coerente e única, porém com códigos multidimensionais (Baddeley, 2000). A capacidade de cada componente da memória operacional aumenta linearmente da infância até o início da adolescência (Case, Kurland, \& Goldeberg, 1982; Gathercole \& Alloway, 2004; Santos \& Bueno, 2003; Santos, Mello, Bueno, \& Dellatolas, 2005). 
A Avaliação Automatizada da Memória Operacional ([AWMA] Automated Working Memory Assessment, Alloway, 2007) foi desenvolvida na língua inglesa tomando como base o modelo de memória operacional de Baddeley e Hitch (1974), considerando as demandas operacionais tanto de armazenamento temporário quanto de processamento de informações em diferentes domínios. Na bateria, estas demandas operacionais são referidas como memória de curto prazo e memória operacional, respectivamente, e representadas por tarefas verbais e visuoespaciais.

A AWMA foi adaptada para a língua portuguesa (Santos \& Engel, 2008) e o desempenho de crianças brasileiras em subtestes verbais não foi influenciado pelo nível socioeconômico (Engel, Santos, \& Gathercole, 2008). A vantagem da utilização de testes validados internacionalmente é a possibilidade de identificar características linguísticas, culturais e fatores pedagógicos que podem influenciar o desenvolvimento dos componentes da memória operacional.

A memória operacional possui um papel importante na aprendizagem durante os anos escolares. Limitações em sua capacidade podem resultar em dificuldades para leitura (Gathercole, Alloway, Willis, \& Adams, 2006; Gathercole, Lamont, \& Alloway, 2006; Siegel \& Ryan, 1989; Swanson, 2003), matemática (Bull \& Scerif, 2001; Geary, Hoard, Byrd Craven, \& DeSoto, 2004; Gersten, Jordan, \& Flojo, 2005), linguagem (Archibald \& Gathercole, 2006; Ellis Weismer et al., 2000; Montgomery, 2000), e atenção (Barkley, 1997; Martinussen \& Tannock, 2006). Portanto, a avaliação dos componentes da memória operacional pode fornecer entendimentos cruciais sobre transtornos de aprendizagem (Gathercole \& Alloway, 2004).

Uma possibilidade de reabilitação da memória operacional seria o treino musical, isto é, o ensino de habilidades musicais por meio da prática regular monitorada, pois alguns estudos sugerem que música, linguagem, leitura e matemática compartilham algumas propriedades acústicas como altura, ritmo e timbre, sendo assim as crianças em ensino musical podem aprender esses conceitos mais prontamente (Anvari, Trainor, Woodside, \& Levy, 2002; Cutietta, 1996a, 1996b; Ilari, 2005). Além disso, há estudos que demonstram a interação entre conhecimentos musicais e a memória operacional em tarefas relacionadas à span de dígitos e memória visuo-espacial (Blundell, Jones, \& Kviklyte, 2007; Fujioka, Ross, Kakigi, Pantev, \& Trainor, 2006; Ho, Cheung, \& Chan, 2003; Parbery-Clark, Skoe, Lam, \& Kraus, 2009; Rauscher \& Zupan, 2000).

Os sons musicais e todos os outros sons compartilham a maioria das etapas de transformação ao longo do eixo neural auditivo (Peretz \& Zatorre, 2005). Por esta razão é necessário que o sistema auditivo integre os sons sequencialmente. Este processo pode ser considerado um mecanismo da memória operacional, que armazena temporária e espacialmente unidades auditivas, combinando-as em um único percepto (Gromko \& Poorman, 1998; Jäncke, 2008; Mallory \& Philbrick, 1995; Rauscher, Shaw, Levine, Ky, \& Wright, 1994; Rauscher et al., 1997).
Platel et al. (1997) demonstraram que a criação de uma imagem mental visual é utilizada como estratégia em tarefas musicais. Portanto, estas evidências sugerem o envolvimento tanto de componentes verbais quanto visuoespaciais da memória operacional no desempenho de atividades musicais.

A complexidade musical é demonstrada em sua execução: o indivíduo deve coordenar a mão exigida e movimentar os dedos na sequência correta. $O$ processo de desempenho musical se realiza visual e auditivamente e possui feedback de integração somatossensorial (Lotze, Scheler, Tan, Braun, \& Birbaumer, 2003). Além desses processos, os músicos devem diferenciar sequências organizadas acusticamente como números de signos limitados, com regras especiais e suas infinitas possibilidades de combinação, por isso a contribuição da memória operacional para o processamento musical é crucial (Peretz \& Zatorre, 2005; Rauscher \& Zupan, 2000; Sallat \& Stachowiak, 2005).

Com os avanços dos estudos neuropsicológicos verificou-se que nos adultos o ritmo e a melodia, que formam as bases da organização musical, são domínios regulados por diferentes hemisférios cerebrais, sendo o hemisfério direito aparentemente mais sensível à melodia e o esquerdo ao ritmo (Zatorre, 2001). Segundo Overy et al. (2004), a especialização hemisférica para o processamento de melodia e ritmo se desenvolve com a idade.

A memória operacional possui um papel importante na representação verbal precisa de sequências rítmicas (Penhune, Zatorre, \& Feindel, 1999) e estas sequências ativam áreas da região do córtex pré-frontal relacionadas à memória operacional (Marin \& Perry, 1999; Zatorre, Belin, \& Penhune, 2002; Zatorre, Evans, \& Meyer, 1994; Zatorre, Evans, Meyer, \& Gjedde, 1992; Zatorre \& Samson, 1991). De acordo com Musacchia, Sams, Skoe e Kraus (2007) o treino musical modificaria a organização cortical, que pode se estender às estruturas sensoriais subcorticais e alcançar locais referentes ao processa-mento da fala, aumentando nos músicos o controle do tronco encefálico tanto para estímulos auditivos como audiovisuais.

O estudo realizado por Rauscher e Zupan (2000) demonstrou que o efeito do treino musical já pode ser constatado nos primeiros meses em tarefas que envolvam a ativação do lobo temporal, conduzindo a uma maior aprendizagem de conceitos específicos como: proporções e frações (Graziano, Peterson, \& Shaw, 1999). O estudo realizado por Rauscher et al. (1997), com crianças pré-escolares de 4 a 6 anos de idade, verificou que o treino musical produz mudanças duradouras em circuitos neurais fundamentais em regiões que não concernem primariamente com a música.

Schmithorst e Holland (2003) demonstraram que o treino musical está associado com o aumento da ativação no giro fusiforme e no córtex pré-frontal do hemisfério esquerdo e interpretaram este achado como uma evidência de ligação entre o treino musical e a memória operacional. Para outros autores o treino musical estaria associado com a eficiência da memória operacional, 
Ribeiro, F. S. \& Santos, F. H. (2012). Treino Musical e Capacidade da Memória Operacional em Crianças Iniciantes, Veteranas e Sem Conhecimentos Musicais.

e também ao aumento das representações abstratas de quantidades numéricas (Williamson, Baddeley, \& Hitch, 2006). Franklin et al. (2008) verificaram que os músicos profissionais exibem escores mais altos do que não músicos em tarefas verbais de span, sugerindo que o treino musical aumenta a capacidade de memória operacional fonológica em adultos.

Conforme os estudos mencionados, a memória operacional e o treino musical estão associadas à ativação de áreas cerebrais recíprocas e homólogas. Contudo não foram encontrados estudos que investiguem se o treino musical pode ampliar a capacidade dos diferentes componentes da memória operacional na infância. O objetivo do presente estudo foi investigar o efeito do treino musical na capacidade da memória operacional em crianças iniciantes e veteranas no treino musical, bem como em um grupo de crianças sem experiência musical. Os objetivos específicos foram: (a) comparar o desempenho de crianças iniciantes e veteranas no treino musical e crianças sem experiência musical em tarefas de estruturação rítmica e memória operacional; (b) identificar quais componentes da memória operacional estão relacionados ao desempenho musical em crianças inseridas no treino musical. As autoras hipotetizam que as crianças veteranas do treino musical alcançarão melhor desempenho nas tarefas de memória operacional do que os demais grupos.

\section{Método}

\section{Aspectos Éticos}

O estudo foi aprovado pelo Comitê de Ética da Universidade Estadual Paulista "Júlio de Mesquita Filho" (UNESP), campus de Assis, processo n ${ }^{\circ}$ 724/2008.

\section{Participantes}

Participaram do estudo crianças iniciantes $(n=20)$, isto é, recém inseridas no treino musical [com conteúdos básicos para discriminar e perceber três dos elementos musicais: duração (o tempo de produção do som), altura do som (grave, médio e agudo) e o timbre (qualidade do som, representado por meio de atividades lúdicas)]; crianças veteranas $(n=20)$ com um ano de treino musical [estimuladas com conteúdos para discriminar e perceber quatro elementos musicais: intensidade (som mais fraco ou mais forte), com o aperfeiçoamento dos conceitos como duração, altura do som e timbre, voltados à escrita e solfejo do que é escutado]; e crianças sem experiência prévia em treino musical para compor o Grupo Controle $(n=20)$. As crianças possuíam idades entre 9 e 10 anos, 36 meninos $(60 \%)$ e 24 meninas $(40 \%)$, nível intelectual médio verificado pelo percentil médio de 62,3 $(D P=18,9)$, conforme Matrizes Coloridas de Raven (Angelini, Alves, Custódio, Duarte, \& Duarte, 1999), e nível socioeconômico médio de 23,6 $(D P=3,8)$, de acordo com Critério de Classificação Econômica Brasil (ABEP, 2008). Estavam matriculadas no $5^{\circ}$ ano e $6^{\circ}$ ano do ensino fundamental de escolas públicas dos municípios de Assis e Ourinhos do Estado de São Paulo. Nenhum dos participantes possuía diagnóstico neuro-lógico e/ou psiquiátrico e nem de deficiência auditiva, histórico de reprovação escolar ou fazia uso de substâncias psicoativas, aferidos por anamnese.

A Tabela 1 apresenta as características amostrais comparadas entre os grupos de crianças iniciantes, veteranas no treino musical e grupo controle, incluindo a idade em meses, escores na capacidade edutiva, Escala Global de Qualidade de Vida e nível socioeconômico. Conforme se percebe, os grupos não diferiram significativamente para quaisquer dessas características.

Tabela 1

Características Amostrais dos Grupos Estudados

\begin{tabular}{lccccc}
\hline & $\begin{array}{c}\text { Iniciantes } \\
(n=20)\end{array}$ & $\begin{array}{c}\text { Veteranos } \\
(n=20)\end{array}$ & $\begin{array}{c}\text { Controles } \\
(n=20)\end{array}$ & $F_{(\mathrm{gl1}, \mathrm{g} 12)}$ & $p$ \\
\hline $\mathrm{N}^{\mathrm{o}}$ de meninos (\%) & $9(45 \%)$ & $14(70 \%)$ & $13(65 \%)$ & $1,45_{(2,57)}$ & 0,24 \\
Idade (meses) & $121,9(9,9)$ & $124,7(8,7)$ & $123,9(7,0)$ & $0,644_{(2,57)}$ & 0,52 \\
Matrizes progressivas de Raven & $69,5(16,1)$ & $59,2(21,6)$ & $59,2(19,0)$ & $1,64_{(2,57)}$ & 0,20 \\
Escala global de qualidade de vida & $21,2(1,1)$ & $21,2(1,7)$ & $19,0(2,7)$ & $0,89_{(2,57)}$ & 0,41 \\
Critério de Classificação Econômica Brasil & $25,2(5,6)$ & $24,2(5,4)$ & $21,5(0,5)$ & $2,20_{(2,57)}$ & 0,12 \\
\hline
\end{tabular}

\section{Materiais}

Anamnese. Desenvolvida por Santos (2002), consiste de um roteiro de perguntas para avaliar aspectos gerais e específicos do desenvolvimento da criança nas dimensões: social, educacional, psicológica e de saúde. Foi utilizada para o rastreio dos critérios de exclusão: presença de reprovação escolar, diagnóstico neurológico, psiquiátrico ou de Transtornos do Desenvolvimento Psicológico e uso crônico de substâncias psicoativas.
Critério de Classificação Econômica Brasil da Associação Brasileira de Empresas de Pesquisas (ABEP, 2008). Escala para avaliar o status socioeconô-mico da família da criança.

Escala Global de Qualidade de Vida ([EGQV], Casas Fernandez, 1997). Constituída de 5 perguntas relacionadas ao comportamento, aprendizagem, autonomia, relações sociais e qualidade de vida. Cada pergunta oferece de 4 a 5 alternativas de respostas indicando do menor para o maior grau de autonomia. 
Matrizes Progressivas Coloridas de Raven - Escala Especial (Angelini et al., 1999). É um teste padronizado que avalia a capacidade edutiva de crianças, constituído por três séries de doze itens (A, AB e B), que aumentam progressivamente em dificuldade. $O$ teste foi utilizado na forma de caderno com gravuras coloridas. Cada item apresenta uma figura incompleta e seis opções de resposta. A criança deve escolher apenas uma das alternativas para completar a figura.

Prova de Ritmos (Stambak, 1968). Este teste consiste em três provas que fornecem uma pontuação total e visam avaliar: (a) reprodução de estruturas rítmicas: o examinador, após ocultar sua mão com um anteparo, reproduz as estruturas rítmicas estabelecidas batendo levemente um lápis contra a mesa e a criança deve identificar a cadência da duração (tempos longos ou curtos) e assim repetir a sequência igualmente; (b) compreensão do simbolismo das estruturas rítmicas: o examinador mostra uma folha de papel com as representações das estruturas rítmicas (por exemplo, $[\cdot \cdot]$ e $[\cdot \cdot]$ ), e pede para a criança diferenciar os símbolos por meio da reprodução sem explicação previa de como reproduzi-las; (c) reprodução: somente após a compreensão do simbolismo das estruturas rítmicas a criança é convidada a bater as 12 primeiras estruturas a partir da visualização das representações das estruturas rítmicas sonoras na folha. O examinador deve anotar a qualidade da estrutura reproduzida (Stambak, 1968).

Teste de Repetição de Pseudopalavras para Crianças Brasileiras (Brazilian Children's Test of Pseudoword Repetition [BCPR], Santos \& Bueno, 2003). O teste consiste na repetição de palavras que podem ser lidas e escritas, porém não possuem significado no idioma (pseudopalavras). A criança deve repetir a pseudopalavra imediatamente após ouvi-la, e assim sucessivamente até o término dos 40 estímulos. O escore é determinado pelo número de repetições corretas. Este teste avalia a capacidade da alça fonológica. Para este estudo foi utilizada a versão computadorizada (Engel et al., 2008).

Avaliação Automatizada de Memória Operacional (Automated Working Memory Assessment [AWMA], Alloway, 2007), versão em Português [reproduzido com permissão de Pearson, Assessment (Direitos Autorais (C) 2007)]. É um teste computadorizado que abrange a avaliação tanto da capacidade de armazenamento quanto de processamento de informações, operações avaliadas de forma balanceada por tarefas na modalidade verbal e visuoespacial. A bateria possui doze subtestes, cada um composto por um número variado de séries, com sequências que aumentam a complexidade progressivamente. Cada série tem o total de seis tentativas, com exceção do subteste que possui somente uma tentativa. Com quatro acertos na mesma série, o programa transfere o examinando automaticamente para o próximo nível de complexidade. Em contraposição, com três erros há a interrupção e consequente transferência para o subteste seguinte. A AWMA foi adaptada junto ao Labo- ratório de Neuropsicologia da UNESP/Assis (Santos \& Engel, 2008). Os subtestes são:

1.Recordação de Dígitos: a criança escuta uma sequência de dígitos e deve lembrar cada sequência na ordem correta.

2.Matriz de Pontos: a criança vê a posição de um ponto vermelho em uma grade quadriculada e deve se lembrar da posição e apontá-la na tela do computador na ordem correta.

3.Julgamento de Frases: a criança escuta uma série de sentenças e deve, além de julgar se cada sentença é verdadeira ou falsa, memorizar a última palavra de cada frase na ordem em que as sentenças foram apresentadas.

4.Discriminação de Formas: a criança vê três figuras, lado a lado, sendo que uma difere das outras, e deve identificar a figura de formato diferente, em seguida deve lembrar a posição em que a figura diferente estava.

5.Recordação de Palavras: a criança escuta uma sequência de palavras e deve lembrar-se de cada sequência na ordem correta.

6.Memória para Labirintos: a criança vê um labirinto com uma trajetória desenhada em vermelho e deve refazer a tragetória no labirinto em branco na tela do computador.

7.Recordação de Contagem: a criança deve contar o número de círculos em um arranjo de círculos e triângulos e depois se lembrar, na ordem correta, o numero de círculos de cada grupo.

8.Julgamento Espacial: a criança vê a figura de dois meninos e identifica se o menino com o chapéu azul está segurando a bola na mesma mão que o menino com o chapéu amarelo; a seguir deve apontar a localização espacial da bola do menino com o chapéu azul.

9.Recordação de Pseudopalavras: a criança escuta uma sequência de pseudopalavras monossílabas e deve se lembrar de cada sequência na ordem correta (este subteste fornece o span de pseudopalavras).

10.Recordação de Blocos: a criança vê uma serie de blocos, numa sequência, e deve reproduzir na ordem correta apontando nos blocos em branco.

11.Recordação de Dígitos Inversos: a criança escuta uma sequência de dígitos e deve lembrar-se de cada sequência na ordem contraria a apresentada.

12.Span Espacial: a criança vê duas formas iguais, mas a forma à direita poderá estar invertida, e deve identificar se a rotação desta forma está do mesmo lado ou ao lado contrário da forma da esquerda; em seguida deve apontar a localização do ponto vermelho acima da figura à direita, em função de sua rotação.

\section{Procedimentos}

As crianças foram selecionadas por meio de reunião escolar com os pais/responsáveis para explicação dos objetivos e procedimentos do estudo. Durante a reunião, 
Ribeiro, F. S. \& Santos, F. H. (2012). Treino Musical e Capacidade da Memória Operacional em Crianças Iniciantes, Veteranas e Sem Conhecimentos Musicais.

os pais assinaram o Termo de consentimento livre e esclarecido e responderam a questões de aspectos socioeconômicos e de qualidade de vida. Após esta etapa, as crianças iniciantes e veteranas em treino musical foram avaliadas individualmente na Escola Municipal de Música de Ourinhos, enquanto que as crianças do grupo controle foram avaliadas na escola Prof ${ }^{a}$ Maria Amélia de Castro Burali. As crianças foram avaliadas pelos seguintes instrumentos: Matrizes Progressivas Coloridas de Raven - Escala Especial, Prova de Ritmos, BCPR e AWMA. As avaliações duraram em média 90 minutos cada com intervalos de 10 minutos quando necessário para evitar o efeito da fadiga. Os instrumentos foram apresentados em ordem semi-aleatória, intercalando tarefas verbais e não verbais.

\section{Resultados}

Os dados obtidos foram analisados segundo o software do “STATISTICA for Windows Edição 99" (Statsoft, 1999).

A Tabela 2 apresenta os resultados das análises de variância de uma via (ANOVA) para verificar a significância dos efeitos da variável independente (grupos: iniciante, veterano e controle) sobre as variáveis dependentes (Total da provas de ritmos, e total do BCPR). Não foram observados efeitos de grupo para a Prova de Rit$\operatorname{mos}[F(2,57)=1,09 ; p<0,34]$ e nem para o escore total do BCPR, isto é, número máximo de acertos $[F(2,57)=$ $2,03 ; p=0,14]$.

Explorando outros aspectos do BCPR, a análise de variância multivariada (MANOVA) não identificou efeito de grupo para o BCPR considerando a extensão dos estímulos, isto é, no desempenho de pseudopalavras de 2, 3, 4 e 5 sílabas $[R$ de $\operatorname{Rao}(8,108)=1,55 ; p=0,14]$. Contudo em relação ao efeito "wordlikeness", isto é, à semelhança das pseudopalavras com palavras - classificada como alta, média e baixa - houve efeito de grupo $[R$ de $\operatorname{Rao}(6,110)=2,58 ; p=0,02]$, confirmado pelo teste post-hoc de Tukey, em que veteranos repetiram mais corretamente as pseudopalavras de alta semelhança com palavras do que os controles $(p=0,05)$. Os controles repetiram melhor pseudopalavras de baixa semelhança com palavras do que os iniciantes $(p=0,03)$. Não houve diferenças com relação às pseudopalavras de média semelhança.

Para a AWMA foi encontrado efeito de grupo por meio de MANOVA $[R$ de $\operatorname{Rao}(24,92)=2,42 ; p=0,001]$ e o teste post-hoc de Tukey demonstrou diferenças nos seguintes subtestes da AWMA: Recordação de Dígitos em que o grupo controle obteve escores abaixo do grupo iniciantes $(p=0,01)$ e em relação aos veteranos $(p=$ $0,001)$. Para a tarefa Matriz de Pontos, os controles obtiveram escores mais baixos que os veteranos $(p=0,03) \mathrm{e}$ os iniciantes $(p=0,05)$. Em Memória para Labirintos os veteranos desempenharam melhor do que os controles ( $p$ $=0,001)$. Em Recordação de Pseudopalavras os veteranos pontuaram mais do que os iniciantes $(p=0,05)$ e con- troles $(p=0,004)$. No subteste Recordação de Dígitos Inversos, tantos os iniciantes $(p=0,01)$ quanto os veteranos $(p=0,04)$ foram superiores aos controles.

A análise do tamanho do efeito calculado por um software de distribuição livre Effect Size Generator for Windows 4.0 (Devilly, 2005) comparou pares de médias obtidas pelos grupos controle, iniciantes e veteranos nos subtestes da AWMA. Foram encontrados efeitos de magnitude média para comparação entre os grupos de iniciantes e veteranos nos subtestes: Discriminação de Formas $(d=0,7)$, Memória para Labirintos $(d=0,7) \mathrm{e}$ Recordação de Pseudopalavras $(d=0,6)$. Foram observados efeitos de magnitude média para comparação entre os grupos iniciante e controle nos subtestes: Matriz de Pontos $(d=0,7)$ e Recordação de Contagem $(d=$ $0,6)$, e de grande magnitude para Recordação de Dígitos $(d=1,1)$ em que as crianças iniciantes obtiveram melhor desempenho, contudo o grupo controle obteve melhor desempenho no subteste Span Espacial $(d=$ $1,0)$. Na comparação realizada entre o grupo veterano e controle foram encontrados efeitos de magnitude média nos subtestes: Matriz de Pontos $(d=0,7)$ Julgamento de Frases $(d=0,6)$, Discriminação de Formas $(d=0,6)$ e de grande magnitude para os subtestes: Memória para Labirintos $(d=1,0)$ e Recordação de Pseudopalavras $(d=1,2)$.

Foram realizadas análises de correlação de Pearson com os grupos iniciante, veterano e controles, com intuito de investigar as associações entre os subtestes do AWMA, BCPR e teste de Ritmos. Somente as correlações $\operatorname{com} p<$ 0,05 serão apresentadas. Foram observadas correlações positivas baixas e moderadas entre alguns subtestes da AWMA e as demais tarefas cognitivas: Recordação de Dígitos se correlacionou com BCPR 2 sílabas $(r=0,27)$, BCPR 5 sílabas $(r=0,26)$ e Prova de Ritmos total $(r=$ $0,48)$. Matriz de Pontos se correlacionou com a Prova de Ritmos total $(r=0,39)$. Julgamento de Frases com BCPR 2 sílabas $(r=0,30)$, com BCPR 5 sílabas $(r=0,32)$, com BCPR baixa semelhança $(r=0,25)$, BCPR total $(r=0,28)$ e Prova de Ritmos total $(r=0,31)$. Recordação de Palavras se correlacionou com BCPR 5 sílabas $(r=0,28)$, BCPR baixa semelhança $(r=0,36)$. Recordação de Pseudopalavras com a Prova de Ritmos total $(r=0,29)$. Recordação de Dígitos Inversos com a Prova de Ritmos total $(r=0,34)$. Não foram observadas correlações entre os demais subtestes da AWMA. Também foram observadas correlações positivas baixas e moderadas entre o BCPR e a Prova de Ritmos: BCPR 2 sílabas $(r=0,32)$, BCPR 5 sílabas $(r=0,41)$, BCPR alta semelhança $(r=$ $0,27)$ e BCPR média semelhança $(r=0,32)$ e BCPR total $(r=0,33)$. Não foram observadas correlações entre os demais subtestes do BCPR e a Prova de Ritmos.

\section{Discussão}

O objetivo do estudo foi investigar o efeito do treino musical na capacidade da Memória Operacional em 
Tabela 2

Comparação entre os grupos iniciantes, veteranos e controle em relação ao total da Prova de Ritmos, do BCPR e dos subtestes da AWMA

\begin{tabular}{|c|c|c|c|c|c|}
\hline Testes & $\begin{array}{l}\text { Iniciantes } \\
(n=20)\end{array}$ & $\begin{array}{l}\text { Veteranos } \\
(n=20)\end{array}$ & $\begin{array}{l}\text { Controles } \\
(n=20)\end{array}$ & $F_{(\mathrm{gl} 1, \mathrm{gl} 2)}$ & $p$ \\
\hline Prova de Ritmos- Total & $16,4(2,8)$ & $17,3(3,0)$ & $15,9(3,5)$ & $1,0_{(2,57)}$ & 0,34 \\
\hline \multicolumn{6}{|l|}{ BCPR } \\
\hline BCPR 2 sílabas & $9,1(1,0)$ & $9,5(0,60)$ & $8,9(0,9)$ & 2,8 & 0,06 \\
\hline BCPR 3 sílabas & $8,3(1,2)$ & $8,7(1,0)$ & $8,7(1,2)$ & 0,7 & 0,47 \\
\hline BCPR 4 sílabas & $8,1(1,6)$ & $8,5(1,0)$ & $8,9(1,1)$ & 1,9 & 0,15 \\
\hline BCPR 5 sílabas & $8,0(1,3)$ & $8,6(1,4)$ & $8,6(1,2)$ & 1,2 & 0,29 \\
\hline BCPR Alta similaridade & $9,1(1,1)$ & $9,5(0,6)$ & $8,8(1,0)$ & 2,8 & 0,06 \\
\hline BCPR Média similaridade & $16,6(1,8)$ & $17,0(1,5)$ & $17,4(1,3)$ & 1,2 & 0,29 \\
\hline BCPR Baixa similaridade & $8,2(1,6)^{\mathrm{a}}$ & $8,9(1,7)^{\mathrm{a}}$ & $9,1(0,7)^{\mathrm{b}}$ & 3,4 & $0,03 *$ \\
\hline BCPR - Total & $33,7(3,8)$ & $35,4(2,1)$ & $35,2(2,3)$ & $2,0_{(2,57)}^{(2,57)}$ & 0,14 \\
\hline \multicolumn{6}{|l|}{ AWMA } \\
\hline Recordação de Dígitos & $26,9(4,1)^{\mathrm{a}}$ & $28,0(5,6)^{\mathrm{b}}$ & $22,7(3,3)^{\mathrm{c}}$ & 7,9 & $0,0008^{*}$ \\
\hline Matriz de Pontos & $23,4(3,8)^{\mathrm{a}}$ & $23,6(5,0)^{\mathrm{a}}$ & $19,9(4,9)^{\mathrm{b}}$ & 4,0 & $0,02 *$ \\
\hline Julgamento de Frases & $10,3(2,7)$ & $11,0(3,0)$ & $9,1(2,7)$ & 2,3 & 0,1 \\
\hline Discriminação de Formas & $11,5(4,7)^{\mathrm{a}}$ & $14,8(4,6)^{b}$ & $11,8(4,5)^{\mathrm{a}}$ & 3,0 & $0,05^{*}$ \\
\hline Recordação de Palavras & $20,6(4,1)$ & $21,5(5,6)$ & $21,6(2,2)$ & 0,3 & 0,73 \\
\hline Memória para Labirintos & $23,0(2,6)^{\mathrm{a}}$ & $25,9(5,0)^{b}$ & $20,6(5,4)^{\mathrm{c}}$ & 7,0 & $0,001 *$ \\
\hline Recordação de Contagem & $14,0(4,8)$ & $13,1(5,8)$ & $14,9(4,5)$ & 0,5 & 0,56 \\
\hline Julgamento Espacial & $7,2(3,0)$ & $8,2(4,0)$ & $7,5(4,7)$ & 0,3 & 0,73 \\
\hline Recordação de Pseudopalavras & $15,4(4,5)^{\mathrm{a}}$ & $18,0(3,0)^{\mathrm{b}}$ & $14,3(2,7)^{\mathrm{c}}$ & 5,7 & $0,005 *$ \\
\hline Recordação de Blocos & $21,4(4,9)$ & $20,5(4,3)$ & $18,4(4,5)$ & 2,1 & 0,12 \\
\hline Recordação de Dígitos Inversos & $13,0(3,1)^{\mathrm{a}}$ & $12,5(4,2)^{\mathrm{a}}$ & $9,7(3,2)^{b}$ & 4,9 & $0,01 *$ \\
\hline Span Espacial & $6,2(2,6)$ & $7,1(6,3)$ & $11,5(5,8)$ & $2,55_{(2,57)}^{(2,57)}$ & 0,09 \\
\hline
\end{tabular}

Nota . Legenda: BCPR = Teste de Repetição de Pseudopalavras para crianças brasileiras; AWMA = Avaliação Automatizada de memória operacional.

* $p<0,05$ no teste post-hoc de Tukey. Obs. Letras iguais indicam que não houve diferenças entre os grupos, letras diferentes indicam diferenças significativas.

crianças de 9 e 10 anos iniciantes ou veteranas no treino musical, em contraste com um grupo de crianças sem experiência prévia de treino musical. $\mathrm{O}$ intuito foi de compreender de que forma o aprendizado musical pode contribuir para o desenvolvimento de habilidades cognitivas utilizando como instrumentos a prova de ritmos, o BCPR e os subtestes da AWMA. Os grupos se mostraram equivalentes em características amostrais, portanto, os fatores socioeconômicos e escolares que poderiam justificar eventuais resultados discrepantes na avaliação neurocognitiva foram controlados.

Não foram detectadas diferenças na Prova de Ritmos entre os grupos, entretanto este teste não avalia características particulares da música como sua escrita ou leitura específica de partitura, demonstrando como citado por Overy et al. (2004) que a especialização hemisférica para o processamento de ritmos se desenvolve com a idade, independente do estudo musical. É importante citar ainda, que a estruturação temporal para execução deste teste é adquirida a partir dos 7 anos, e que no decorrer do desenvolvimento as crianças adquirem a possibilidade de estruturar conjuntos sempre mais longos e complexos (Stambak, 1968).

Este é o primeiro estudo brasileiro utilizando toda a bateria AWMA que avalia os componentes da memória operacional por tarefas computadorizadas nas modalidades verbais e visuoespaciais, tendo identificado diferenças significativas entre os grupos nos subtestes Recordação de Dígitos, Matriz de Pontos, Discriminação de Formas, Memória para Labirintos e Recordação de Pseudopalavras. Nestes, as crianças veteranas do treino musical obtiveram melhor desempenho que os demais grupos. Para Recordação de Dígitos Inversos tanto as crianças iniciantes quanto as veteranas obtiveram maior pontuação do que controles. Tal achado indica que o treino musical pode favorecer melhores resultados tanto em tarefas referentes ao armazenamento de informações (memória de curto prazo verbal e visuoespacial), corroborando com Jäncke (2008), como ao controle de processamento (memória operacional). Estes dados demonstram que tanto o armazenamento das informações quanto o processamento visuoespacial da memória ope- 
Ribeiro, F. S. \& Santos, F. H. (2012). Treino Musical e Capacidade da Memória Operacional em Crianças Iniciantes, Veteranas e Sem Conhecimentos Musicais.

racional participariam diretamente da aprendizagem musical corroborando com estudos prévios (Blundell et al., 2007; Ho et al., 2003; Parbery-Clark et al., 2009; Peretz \& Zatorre, 2005; Rauscher \& Zupan, 2000; Williamson et al., 2006).

Estes dados sugerem que o treino musical pode contribuir no desenvolvimento da capacidade da memória operacional (Fujioka et al., 2006; Schmithorst \& Holland, 2003) e também da orientação espacial (Gromko \& Poorman, 1998; Mallory \& Philbrick, 1995; Rauscher et al., 1994; Rauscher et al., 1997; Rauscher \& Zupan, 2000). As crianças em treino musical lidam principalmente com imagens, sendo que desde o início do programa desenvolvem a percepção rítmica relacionada ao tempo de produção do som, que é por sua vez fortalecida na segunda fase, quando se tornam veteranas junto ao programa de treino musical.

No subteste de Recordação de pseudopalavras da AWMA que avalia o span no sentido clássico, ou seja, por meio de uma sobrecarga do sistema a partir da memorização de um número crescente de estímulos, as crianças veteranas obtiveram maior pontuação que os iniciantes e controles. Isso representa uma maior capacidade da memória operacional fonológica, corroborando com Franklin et al. (2008).

No Teste de Repetição de Pseudopalavras para crianças brasileiras (BCPR), não houve diferenças entre os grupos quanto ao escore total ou por extensão de sílabas. Os escores obtidos no BCPR se encontravam dentro da média esperada para a idade, sendo que o aumento na extensão de sílabas das pseudopalavras do BCPR representa um incremento na carga de memorização, isto é, a capacidade do sistema (Santos \& Bueno, 2003). Por outro lado, quando foi considerada a semelhança com pseudopalavras, o efeito de grupo foi observado, demonstrando que pseudopalavras mais semelhantes às palavras foram repetidas mais corretamente por veteranos do que por controles. Este dado indica que crianças veteranas em treino musical processam melhor sons familiares (Musacchia et al., 2007).

$\mathrm{O}$ resultado divergente entre BCPR e o subteste repetição de pseudopalavras da AWMA pode ser explicado pela natureza menos complexa do primeiro, pois neste as pseudopalavras são apresentadas uma a uma e possuem de duas a cinco sílabas. No subteste da AWMA todas as pseudopalavras são monossílabas ou dissílabas, porém apresentadas em séries de extensões que vão de um a oito estímulos por vez.

Os subtestes da bateria AWMA (Matriz de pontos, Recordação de Dígitos e Recordação de Dígitos inversos) apresentaram correlações positivas com o resultado total da Prova de Ritmos, corroborando a relação entre o ritmo e armazenamento visuoespacial, e também entre o ritmo e memória operacional verbal (Marin \& Perry, 1999; Zatorre et al., 2002; Zatorre et al., 1994; Zatorre et al., 1992; Zatorre \& Samson, 1991). Isto significa que a memória operacional possui um papel importante na represen- tação fonológica precisa em sequências rítmicas (Penhune et al., 1999). E estas sequências rítmicas ainda ativariam áreas relativas à memória operacional (Marin \& Perry, 1999; Zatorre et al., 2002; Zatorre et al., 1994; Zatorre et al., 1992; Zatorre \& Samson, 1991) e, consequentemente, o julgamento espacial que envolve a propriedade visuoespacial (Rauscher et al., 1994). Cabe mencionar que apenas uma criança do grupo iniciante e outra do grupo controle conseguiram fazer todas as sequências de ritmos enquanto que quatro crianças do grupo de veteranos acertaram todas as sequências rítmicas.

Outros subtestes da AWMA como Recordação de Dígitos, Julgamento de Frases e Recordação de Palavras apresentaram correlações positivas com os resultados do BCPR, resultado que corrobora a validade de constructo, conforme os estudos de Gathercole (1994) e Santos e Bueno (2003). Isso indicaria que quanto melhor o desempenho em testes relacionados à memória operacional verbal, maior capacidade da alça fonológica. Portanto uma explicação plausível para este resultado seria que o aluno que está inserido no treino musical desenvolveria representações fonológicas mais precisas, específicas e categorizadas quanto ao processamento fonológico.

Não houve correlação entre os testes Repetição de Pseudopalavras da bateria AWMA e BCPR; contudo, o fato de que ambos os testes se correlacionaram com as provas de ritmos sugere que provavelmente a ausência de associação entre estes seja devida à menor complexidade relacionada às pseudopalavras de baixa similaridade com palavras que compreendem apenas 10 dos 40 estímulos do teste.

Outra explicação plausível para esta incongruência, se refere ao sistema de pontuação intrínseco ao instrumento usado (Santos et al 2012). Por exemplo, a AWMA considera além do span, a consistência de respostas, isto é, o número máximo de seqüências corretas de uma dada extensão. Isto significa que duas crianças podem ter um span de três, ou seja, capacidade para memorizar precisamente até três pseudopalavras, entretanto se uma delas recordar quatro seqüências consecutivas receberá uma pontuação maior do que a outra que recorde menos seqüências contendo pseudopalavras de mesma extensão. Portanto, esta particularidade da AWMA pode constituir um artefato.

Uma das limitações deste estudo foi o pequeno número de crianças participantes, porém as variáveis relevantes que poderiam confundir os resultados foram controladas. Considerada esta limitação, os resultados do presente estudo sugerem que o treino musical potencializa a capacidade da memória operacional e contribue para a compreensão dos benefícios inerentes à experiência musical, a partir do ensino da música. Contudo, são necessários estudos longitudinais - como o que se encontra em desenvolvimento junto ao Laboratório de Neuropsicologia da UNESP/Assis - para a investigação dos efeitos do treino musical sobre o desenvolvimento cognitivo e sua contribuição para o bem-estar social e acadêmico das crianças. 
Em conclusão, conforme a hipótese a priori, o grupo de crianças veteranas no treino musical obteve melhores escores em tarefas cognitivas referentes à memória operacional (visuoespacial e verbal). Entretanto, no presente estudo não é possível discriminar se este ganho foi igual ou superior ao desenvolvimentoassociado à idade.

\section{Referências}

Alloway, T. P. (2007). Automated Working Memory Assessment. London: Harcourt Assessment.

Angelini, A. L., Alves, I. C. B., Custódio, E. M., Duarte, W. F., \& Duarte, J. L. M. (1999). Matrizes progressivas coloridas de Raven. São Paulo, SP: Centro Editor de Testes e Pesquisas em Psicologia.

Anvari, S. H., Trainor, L. J., Woodside, J., \& Levy, B. A. (2002). Relations among musical skills, phonological processing, and early reading ability in preschool children. Journal of Experimental Child Psycology, 83, 111-120.

Archibald, L. M. D., \& Gathercole. S. E. (2006). Short-term and working memory in children with Specific Language Impairments. International Journal of Language and Communication Disorders, 41, 675-693.

Associação Brasileira de Empresas de Pesquisas. (2008). Critério de classificação econômica no Brasil. Retrieved June 16, 2008, from http://www.abep.org/codigosguias/ ABEP_CCEB.pdf

Baddeley, A. D. (1992). Working memory. Science, 255, 556559.

Baddeley, A. D. (2000).The episodic buffer: A new component of working memory? Trends in Cognitive Sciences, 4, 417423.

Baddeley, A. D., \& Hitch, G. J. (1974). Working memory. In G. A. Bower (Ed.), The psychology of learning and motivation: Advances in research and theory (pp. 47-89). New York: Academic Press.

Barkley, R. A. (1997). ADHD and the nature of self-control. New York: Guilford.

Blundell, J., Jones, S., \& Kviklyte, S. (2007). Performance on neuropsychological measures of executive function between music and non-music students. Paper presented for B.S.c Psychology, University of Wales, Bangor, ME.

Bull, R., \& Scerif, G. (2001). Executive functioning as a predictor of children's mathematics ability, shifting, inhibition and working memory. Developmental Neuropsychology, 19, 273-293.

Casas Fernandez, C. (1997). Experiencia con el cuestionario de calidad de vida en el niño con epilepsia (CAVE). Review of Neurology, 25(139), 415-421.

Case, R. D., Kurland, M., \& Goldberg, J. (1982). Operational efficiency and the growth of short-term memory span. Journal of Experimental Child Psychology, 33, 386-404.

Cutietta, R. A. (1996a). Language and music programs. UPDATE: The applications of Research in Music Education, 9, 26-31.

Cutietta, R. A. (1996b). Does music instruction aid mathematical skills? UPDATE: The applications of Research in Music Education, 9, 28-30.

Devilly, G. J. (2005). The Effect Size Generator for Windows (Version 4.0) [Computer program]. Melbourne, Australia: Brain Sciences Institute.
Ellis Weismer, S., Tomblin, J. B., Zhang, X., Buckwalter, P., Gaura Chynoweth, J., \& Jones, M. (2000). Nonword repetition performance in school-age children with and without language impairment. Journal of Speech, Language, and Hearing Research, 43, 865-878.

Engel, P., Santos, F. H., \& Gathercole, S. E. (2008). Are working memory measures free of socio-economic influence? Journal of Speech, Language and Hearing Research, 51, 1580-1587.

Franklin, M. S., Moore, K. S., Yip, C. Y., Jonides, J., Rattray, K., \& Moher, J. (2008). The effects of musical training on verbal memory. Psychology of Music, 36(3), 353-365.

Fujioka, T., Ross, B., Kakigi, R., Pantev, C., \& Trainor, L. J. (2006). One year of musical training affects development of auditory cortical-evoked fields in young children. Brain, 129, 2593-2608.

Gathercole, S. E. (1994). Neuropsychology and working memory: A review. Neuropsychology, 8, 499-505.

Gathercole, S. E., \& Alloway, T. P. (2004). Working memory and classroom learning. Professional Association for Teachers of Students with Specific Learning Difficulties, 17, 2-12.

Gathercole, S. E., Alloway, T. P., Willis, C., \& Adams, A. (2006). Working memory in children with reading disabilities. Journal of Experimental Child Psychology, 93(3), 265-281.

Gathercole, S. E., Lamont, E., \& Alloway, T. P. (2006). Working memory in the classroom. In S. Pickering (Ed.), Working memory and education. London: Academic Press.

Geary, D. C., Hoard, M. K., Byrd Craven, J., \& DeSoto, M. C. (2004). Strategy choices in simple and complex addition: Contributions of working memory and counting knowledge for children with mathematical disability. Journal of Experimental Child Psychology, 88, 121-151.

Gersten, R., Jordan, N. C., \& Flojo, J. R. (2005). Early identification and interventions for students with mathematics difficulties. Journal of Learning Disabilities, 38, 293-304.

Graziano, A. B., Peterson, M., \& Shaw, G. L. (1999). Enhanced learning of proportional math through music training and spatial-temporal training. Neurological Research, 21(2), 139-152.

Gromko, J. E., \& Poorman, A. S. (1998). The effect of music training on preschooler's spatialtemporal task performance. Journal of Research in Music Education, 46, 173-181.

Ho, Y. C., Cheung, M. C., \& Chan, A. S. (2003). Music training improves verbal but not visual memory: Cross-sectional and longitudinal explorations in children. Neuropsychology, 17, 439-450.

Ilari, B. (2005). Research on music, the brain and cognitive development: Addressing some common questions of music educators. Music Education International, 2, 85-97.

Jäncke, L. (2008). Music, memory and emotion. Journal of Biology, 7(6), 1-5.

Lotze, M., Scheler, G., Tan, H. R. M., Braun, C., \& Birbaumera, N. (2003). The musician's brain: Functional imaging of amateurs and professionals during performance and imagery. NeuroImage, 20(3), 1817-1829.

Mallory, M. E., \& Philbrick, K. E. (1995). Music training and spatial skills in children. Paper presented at the meeting of the American Psychological Society, New York.

Marin, O. S. M., \& Perry, D. W. (1999). Neurological aspects of music perception and performance. In D. Deutsch (Ed.), The Psychology of Music (pp. 653-724). San Diego, CA: Academic Press. 
Ribeiro, F. S. \& Santos, F. H. (2012). Treino Musical e Capacidade da Memória Operacional em Crianças Iniciantes, Veteranas e Sem Conhecimentos Musicais.

Martinussen, R., \& Tannock, R. (2006). Working memory impairments in children with attention-deficit hyperactivity disorder with and without comorbid language learning disorders. Journal of Clinical and Experimental Neuropsychology, 28, 1073-1094.

Montgomery, J. (2000). Verbal working memory in sentence comprehension in children with specific language impairment. Journal of Speech, Language, and Hearing Research, 43, 293-308.

Musacchia, G., Sams, M., Skoe, E., \& Kraus, N. (2007). Musicians have enhanced subcortical auditory and audiovisual processing of speech and music. Proceedings of the National Academy of Sciences of the USA, 104, 15894-15898.

Overy, K., Norton, A., Cronin, T., Gaab, N., Alsop, D., Winner, E., et al. (2004). Imaging melody and rhythm processing in young children. Neuroreport Lippincott Williams \& Wilkins, 15, 1723-1726.

Parbery-Clark, A., Skoe, E., \& Kraus, N. (2009). Musical experience limits the degradative effects of background noise on the neural processing of sound. Journal of Neuroscience, 29, 14100-14107.

Penhune, V. B., Zatorre, R. J., \& Feindel, W. H. (1999). The role of auditory cortex in retention of rhythmic patterns in patients with temporal-lobe removals including Heschl's gyrus. Neuropsychologia, 37, 315-331.

Peretz, I., \& Zatorre, R. J. (2005). Brain organization for music processing. Annual Review of Psychology, 56, 89-114.

Platel, H., Price, C., Baron, J.-C., Wise, R., Lambert, J., Frackowiak, R. S. J., et al. (1997). The structural components of music perception- A functional anatomical study. Brain, 120, 229-243.

Rauscher, F. H., Shaw, G. L., Levine, L. J., Ky, K. N., \& Wright, E. L. (1994). Music and spatial task performance: A causal relationship. Paper presented at the meeting of the American Psychological Association, Los Angeles, CA.

Rauscher, F. H., Shaw, G. L., Levine, L. J., Wright, E. L., Dennis, W. R., \& Newcomb, R. L. (1997). Music training causes longterm enhancement of preschool children's spatial-temporal reasoning. Neurological Research, 19(1), 1-8.

Rauscher, F. H., \& Zupan, M. A. (2000). Classroom Keyboard Instruction Improves Kindergarten Children's Spatial-Temporal Performance: A field experiment. Early Childhood Research Quarterly, 15(2), 215-228.

Repovš, G., \& Baddeley, A. (2006). The multi-component model of working memory: Explorations in Experimental Cognitive Psychology. Neuroscience, 139, 5-21.

Sallat, S., \& Stachowiak, F. J. (2005). Working memory for music and language: Comparison of normal and language impaired 5-year old children. Poster-Präsentation auf dem Kongress "The neurosciences and music II", University of Gießen, Justus, Liebig, Leipzig, Germany.

Santos, F. H. (2002). Memória operacional de crianças normais e com lesões congênitas: Desenvolvimento cognitivo e reorganização cerebral. (Tese de Doutorado não-publicada). Universidade Federal de São Paulo, SP.

Santos, F. H., \& Bueno, O. F. A. (2003). Validation of the Brazilian Children's Test of Pseudoword Repetition in Portuguese speakers aged 4 to 10 years. Brazilian Journal of Medical and Biological Research, 36(11), 1533-1547.
Santos, F. H., \& Engel, P. M. J. (2008). Adaptação Brasileira da AWMA: "Automated Working Memory Assessment". In K. Z. Ortiz, L. Mendonça, A. Foz, C. B. Santos, D. Fuentes, \& D. A. Azambuja (Ed.), Avaliação neuropsicológica. Panorama interdisciplinar dos estudos atuais na normatização e validação de instrumentos no Brasil (pp. 352-362). São Paulo, SP: Vetor.

Santos, F. H., Mello, C. B., Bueno, O. F., \& Dellatolas, G. (2005). Cross-cultural differences for three visual memory tasks in Brazilian children. Perceptual and Motor Skills, 101, 421-433.

Schmithorst, V. J., \& Holland, S. K. (2003). The effect of musical training on the neural correlates of math processing: A functional magnetic resonance imaging study in humans. Neuroscience Letters, 354(3), 193-196.

Siegel, L. S., \& Ryan, E. B. (1989). The Development of Working Memory in Normally Achieving and Subtypes of Learning Disabled Children. Child Development, 60(4), 973980.

Stambak, M. (1968). Três provas de ritmo. In R. Zazzo (Ed.), Manual para exame psicológico da criança (pp. 107-125). São Paulo, SP: Mestre Jou.

Statsoft. (1999). Statistica for Windows [Computer program manual]. Tulsa, OK: Author.

Swanson, H. L. (2003). Age-related diVerences in learningdisabled and skilled readers' working memory. Journal of Experimental Child Psychology, 85, 1-31.

Williamson, V. J., Baddeley, A. D., \& Hitch, G. J. (2006). Music in working memory? Examining the effect of pitch proximity on the recall performance of nonmusicians. Paper presented at the $9^{\text {th }}$ International Conference on Music Perception and Cognition, Alma Mater Studiorum, University of Bologna, Italy.

Zatorre, R. J. (2001). Neural specializations for tonal processing. In R. J. Zatorre \& I. Peretz (Eds.), The biological foundations of Music (pp. 193-210). New York: NY Academy Science.

Zatorre, R. J., Belin, P., \& Penhune, V. B. (2002). Structure and function of auditory cortex: Music and speech. Trends in Cognitive Sciences, 6, 37-46.

Zatorre, R. J., Evans, A. C., \& Meyer, E. (1994). Neural mechanisms underlying melodic perception and memory for pitch. Journal of Neuroscience, 14, 1908-1919.

Zatorre, R. J., Evans, A. C., Meyer, E., \& Gjedde, A. (1992). Lateralization of phonetic and pitch processing in speech perception. Science, 256, 846-849.

Zatorre, R. J., \& Samson, S. (1991). Role of the right temporal neocortex in retention of pitch in auditory short-term memory. Brain, 114, 2403-2417. 